\title{
Journal of Huntington's Disease
}

These are exciting times for Huntington's disease research. We have seen great progress in recent years, and the revolutions in genomics and molecular biology are providing advances in understanding pathogenesis, such as the discoveries of mutant huntingtin aggregation and inclusion body formation, specific proteolytic cleavage of huntingtin, identification of critical clearance pathways, transcriptional changes that occur early in disease, electrophysiology and synaptic transmission deficits, inflammation, critical post-translational modifications, and aberrant protein interactions, among many other important findings. With the identification of the genetic cause of the disease has also come the ability to model the disease in multiple systems, including more recently in patientderived induced pluripotent stem (iPS) cell models of the disease, to link genetics and neuronal function in Huntington's disease and related disorders. In turn, these advances are beginning to translate into potential future treatments with the development of phosphodiesterase, caspase and KMO inhibitors, novel compounds acting on sirtuin pathways, and huntingtin lowering therapies such as RNAi, zinc-finger agents, and anti-sense oligonucleotides that are now moving towards clinical trials.

Such a wealth of promising research deserves a forum, and as Editors-in-Chief, we are delighted to announce the launch of the Journal of Huntington's Disease (http://www.iospress.nl/journal/journalof-huntingtons-disease/), a new high-quality journal giving equal weight to original research in basic science, translational research and clinical studies in Huntington's disease and related disorders.

The Journal of Huntington's Disease has attracted support from a truly outstanding Editorial Board (http://www.iospress.nl/journal/journal-ofhuntingtons-disease/?tab=editorial-board), which bodes extremely well for the success of the journal. The launch issue features a strong line-up of research articles and reviews. In addition to full research article and reviews, the Journal of Huntington's Disease will publish short communications, invited editorials, letters-to-the-editor and a series of historical perspectives. All articles will be published both online and in print. Moreover, the journal offers rapid publication with an affordable open access option.

We are confident that the Journal of Huntington's Disease will serve the community well and will be an influential and positive force in advancing the frontiers of research on Huntington's disease and related disorders in the coming years and decades.

Editor-in-Chief Blair R. Leavitt University of British Columbia Vancouver, Canada

Editor-in-Chief Leslie M. Thompson University of California Irvine, CA, USA 